\title{
COGNITIVE VISUALIZATION IN A HYBRID INTELLIGENT RISK CONTROLLING SYSTEM
}

\author{
Maria Skvortsova, Valery Terekhov, Valery Chernenky and Ivan Chernenky \\ Bauman Moscow State Technical University, Russia
}

\begin{abstract}
Currently developed hybrid intelligent systems that use a combination of traditional data processing methods and intelligent methods are able to analyze and assess the existing risks in all areas of human activity. This article shows the positive effect of cognitive computer visualization as a means of activating the mechanisms of visual-figurative thinking of the person making a decision in the tasks of controlling the integral indicator of risks. The essence of the anamorphic method is shown, which is the transition from one visual image built on the basis of Euclidean metrics to another visual image based on the quantitative metric of the selected risk. The mathematical formulation of the dynamic meta-anamorphine method is disassembled. The advantages of the constructed meta-anamorphosis in the risk controlling HIS are given. The problem of calculating the integral risk index, which combines the risks of different spheres of activity and visualizations built on this basis, is analyzed. Conclusions are made regarding the construction of a hybrid model based on the hierarchy analysis method and the dynamic meta-anamorphosis method. The directions of further research are determined.
\end{abstract}

\section{KEYWORDS}

Cognitive Visualization, Anamorphosis, Meta-Anamorphosis Method, Neural Network, Genetic Programming

\section{INTRODUCTION}

Recently, the market of hybrid intelligent systems (HIS) of various functions, including risk management HIS, has been intensively developing, as these systems take advantage of various methods and overcome inherent shortcomings of those methods. In addition, HIS can effectively handle formalized and non-formalized knowledge through integration which uses both traditional data processing techniques and artificial intelligence methods - expert and fuzzy methods, artificial neural networks, genetic algorithms, etc.

\section{THEORETICAL JUSTIFICATION}

\subsection{Theoretical Aspects of Safety and Risk Controlling}

One of the most discussed topics in Russia and the world today is the topic of globalization and security of economic, political and cultural processes, including controlling the risks. The emergence of these processes is the growing unification of information and communication technologies, methods of decision-making, the formation of common stereotypes of thinking and behavior of subjects operating in the economic, social, information and cultural space. Objects of increased extra attention are religions, ideologies, systems of standards and international treaties, national and regional currencies, etc. Due to the rapidly changing global environment, the role of the state in the security of the country becomes blurred, as these processes are beginning to be influenced by a large number of factors that the state is not able to quickly track and change. Examining in detail each process and the areas in which it occurs, it is possible to form a list of risks that can lead to a negative or critical situation.

In these circumstances, a particular interest is presented in the study of the types of HIS able to comprehensively analyze and assess the existing risks in the most critical areas of human activity (state, military, political, economic, social, information, technological, environmental, etc.), to create scenarios from 
the current situation and provide recommendations on protection measures. We will call such hybrid intelligent systems risk controlling HIS, where risk is a combination of the probability of damage and the severity of this damage, and risk control - information and analytical support of decision-making processes in the assessment of existing risks in the most critical areas of human activity. In this case, risk analysis is the systematic use of information obtained from all available sources to identify hazards and quantify the risk, and acceptable risk - a risk that in this situation is considered acceptable with the existing social values.

\subsection{Description of the Module "Intellectual Data Analysi" in Risk Controlling}

The generalized structure of HIS risk assessment and the functions of its structure of modules were discussed in detail in (Skvortsova et al., 2017). The peculiarity of the structure of the "Intellectual data analysis" module of the risk controlling HIS under consideration is that in its work it uses one of the methods of cognitive visualization, namely the method of dynamic meta-anamorphosis. In this case, cognitive visualization is defined as data visualization methods that allow to activate visual-figurative mechanisms of thinking of the decision-maker and facilitate the solution of a complex problem.

The choice of the method is caused by the fact that it leads to a sharp simplification and acceleration of the time of complex analysis and risk assessment in the most critical areas of human activity and can be used in risk controlling HIS as one of the methods of analysis of hierarchies, which helps to determine the optimal solution in this situation.

\subsection{The Method of Dynamic Metamorphosis}

The method is based on the concept of anamorphosis, which is defined as the transformation of one visual image built on the basis of Euclidean metrics to another visual image based on the metric of the selected indicator (risk).

In the most general form, the method can be shown as follows. Figure 1 shows a fragment of a map of an area with a square grid applied to it, each cell of which corresponds to a certain indicator of risk (Figure 1a) and its corresponding risk matrix (Figure 1b).

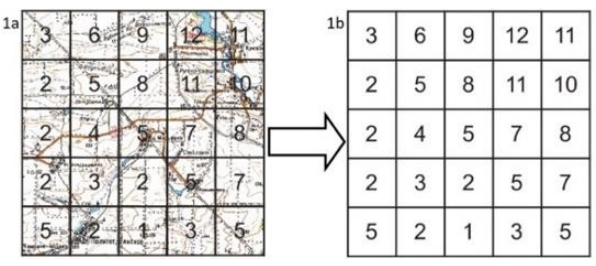

Figure 1. Risk matrix

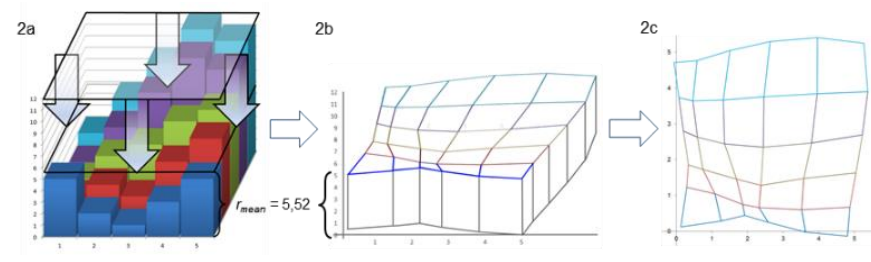

Figure 2. Anamorphosis of a three-dimensional risk matrix

The risk matrix can be represented as a three-dimensional matrix (Figure 2a), where the height of each column corresponds to the risk value in each cell of the original matrix (Figure 2b).

Then the anamorphic operation can be represented as "pressing" on the top side of the matrix to a level that is equal to the average risk value across the matrix (in this specific case $r_{\text {mean }}=5,52$ ).

This makes the columns higher than $r_{\text {mean }}$ shrink to this value while increasing their surfaces, and the columns were below the average value, "catch up" to the level of $r_{\text {mean }}$, decreasing their surfaces (Figure 2b). The result of the operation is a matrix (Figure 2c) in which the risk indicators in all cells are the same and equal to the mean value $r_{\text {mean }}$, which makes it possible to carry out visual modeling of the solution in the linear space of the selected risk index.

A dynamic meta-anamorphosis is an anamorphosis built on the basis of the integral risk index, with each of the risks included in the integral index varying over time.

We consider the mathematical formulation of the problem of the anamorphic algorithm, based on the following considerations.

Let $\boldsymbol{D}$ be an area on the plane $\boldsymbol{R}^{2}$ (an areal figure constructed on the basis of the selected risk), which should be anamorphosed. In this case, the risk distribution is described by the function $p(z)$, a priori defined on the $\boldsymbol{D}$ part of the plane $\left(z=(x, y)-\right.$ a point on the plane $\left.\boldsymbol{R}^{2}\right)$. Without loss of generality, we can assume that the 
function $p(z)$ is defined on the entire plane $\boldsymbol{R}^{2}$. For this purpose, it can be defined as constant outside the region $\boldsymbol{D}$ (mean value $\bar{p}$ of the function $p(z)$ on the region $\boldsymbol{D}$ ). Then the anamorphosis is given by the transformation $h: R^{2} \rightarrow R^{2}(h:(x, y) \mapsto(u, v))$

or functions $U(x, y)$ and $V(x, y)$, where $u=U(x, y), v=V(x, y)$ are continuous, one-to-one functions.

The $h$ transformation changes the area around the point $(x, y)$ with a coefficient

$$
J=J(U, V)=\frac{\partial U}{\partial x} \cdot \frac{\partial V}{\partial y}-\frac{\partial U}{\partial y} \cdot \frac{\partial V}{\partial x} .
$$

The condition $p(x, y)=\bar{p}=$ const may be rewritten as $J(U, V)=p(x, y) / \bar{p}$. Then the problem of finding the anamorphosis is reduced to solving the equation

$$
\frac{\partial U}{\partial x} \cdot \frac{\partial V}{\partial y}-\frac{\partial U}{\partial y} \cdot \frac{\partial V}{\partial x}=\frac{p(x, y)}{\bar{p}},
$$

for which $[U(x, y), V(x, y)]$ defines a one-to-one transformation .

As a result of this transformation, each area shape is deformed according to a given function, which leads to a change in the overall appearance of the entire area $\boldsymbol{D}$.

There are a number of difficulties in the construction of anamorphosis, the overcoming of which is described in detail in (Terekhov et al., 2019; Chernenkiy, V. et al, 2018).

The anamorphic algorithm is an iterative process that stops when the relative deviations of the areas of all $N$ figures become less than the predetermined error value $\varepsilon$.

Since the anamorphic algorithm assumes each cell to be represented in the form of a circle of area $\tilde{s}_{i}=P_{i} / \bar{p}$, it is possible to work with the matrix of integral risk, which takes into account the many risks that are the criteria for solving the problem. In such a matrix, the integral area of each cell will be determined as

$$
\tilde{s}_{i \text { int }}=\left(\tilde{s}_{i 1}+\tilde{s}_{i 2}+\ldots+\tilde{s}_{i R}\right) / R \text {, }
$$

where $R$ is the number of risks under consideration (Figure 3 ).
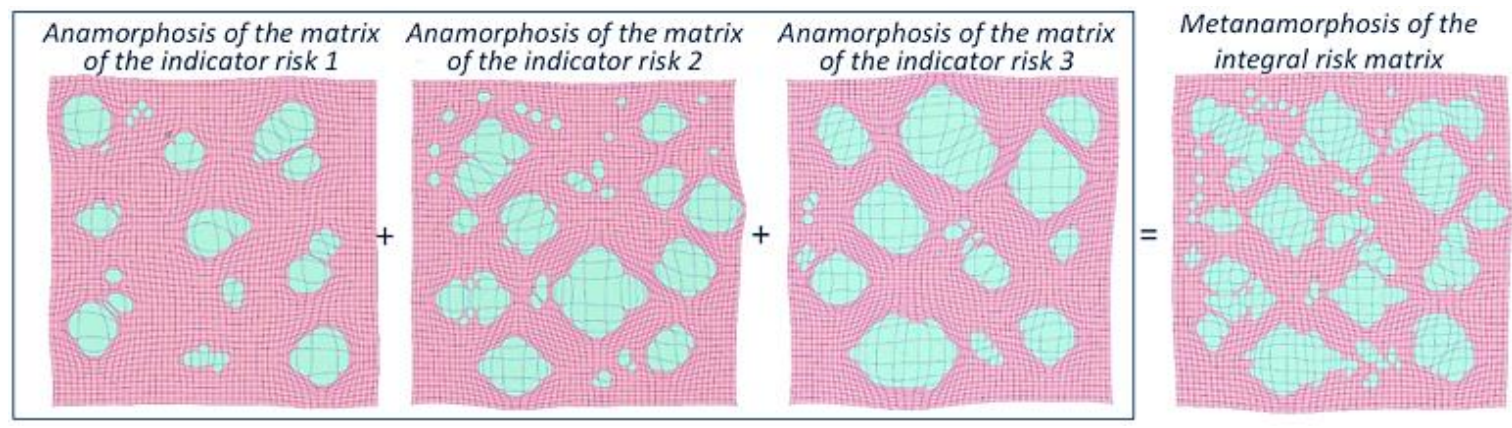

Figure 3. Meta-anamorphosis of integral risk matrix

The blue areas on the anamorphosis of the risk index matrices 1-3 correspond to those areas of the map where the risk is higher than acceptable, and on the meta-anamorphosis of the integral risk matrix they correspond to those areas of the map where the value of the integral risk is higher than acceptable. For simplicity, Figure 3 it is not shown on the map underlay on the basis of which the risk matrices 1-3 were calculated.

This approach allows to move from an anamorphosis calculation based on a single matrix of local risks to a meta-anamorphosis working with a matrix of integral risk.

In the course of research in (Terekhov et al., 2019), a variant of the modified anamorphic algorithm was proposed, in the calculated part of which an artificial neural network with one hidden layer was used, which allows to significantly reduce the operation time of the algorithm with an acceptable loss of computational accuracy. In (Chernenkiy, V. et al, 2018; Terekhov et al., 2019), the method of presentation of risks in the form of time series and their prediction using symbolic regression is presented, which is a method for constructing regression models by trying different random composition of functions from a given set. The implementation of symbolic regression involves the generation of a formula in the form of a syntactic tree, using the method of genetic programming (a special case of a genetic algorithm that works with syntactic trees of variable length).

The work has shown that the obvious advantages of dynamic meta-anamorphosis in solving the problem of analysis, assessment and controlling of risks in the most critical areas of human activity are: 
- reducing the dimension of the problem by the number of local risks reduced into anamorphic integral indicator;

- possibility of visual modeling of solutions, taking into account the local/integral risk changing in time, which allows to determine the negative/positive trends in the change of the given risks and to offer qualitative and quantitative measures for their neutralization to the set values;

- identification of hidden patterns of behavior over time of various parameters of the problem, implicitly dependent on the integral risk index;

- the possibility of constructing scenarios of action of the decision maker based on visual spatio-temporal analysis of dynamic meta-anamorphosis, taking into account the processes associated with the rapid evolution of local or integral risk.

\section{PRACTICAL IMPLEMENTATION}

The software implementation of the considered algorithm allowed to obtain a meta-anamorphosis based on the integral anamorphic index. The following risk matrices were taken as an integral indicator: floods and floods, fire danger and natural conditions for human life. Figure 4 shows a meta-anamorphosis constructed on the basis of integral risk, where the darker shaded cell, the higher it is the integral indicator of risk.

The analysis of the obtained meta-anamorphosis allows to determine the areas of maximum and permissible values of the integral risk and to make decisions on its prevention in a homogeneous linear space of this integral risk.

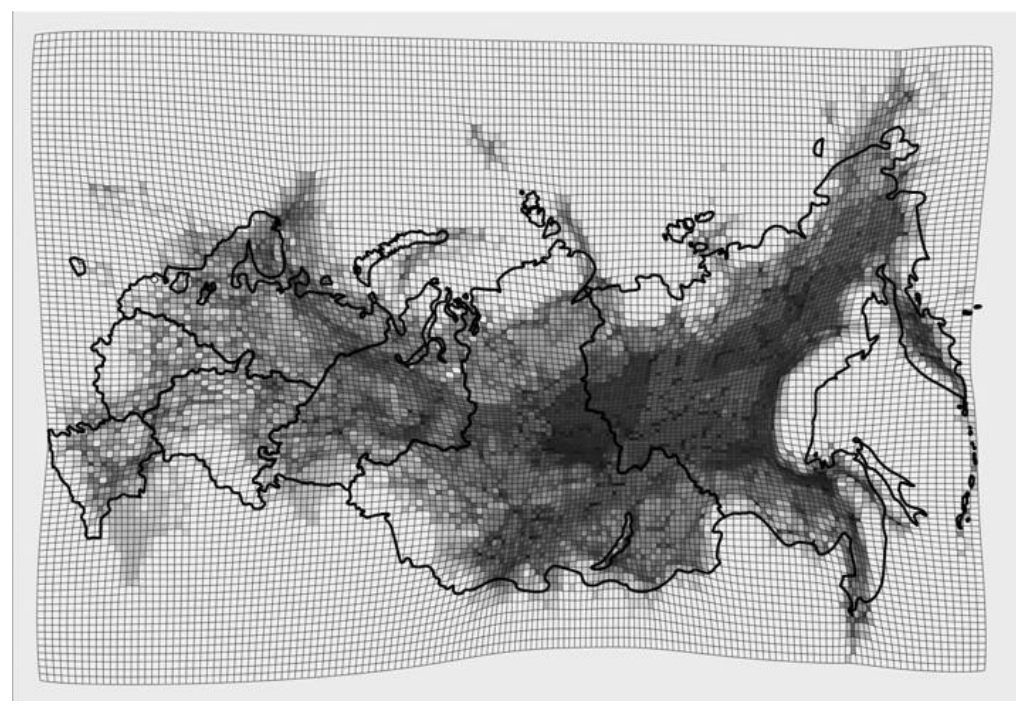

Figure 4. A meta-anamorphosis of integrated flood risk and flooding, fire danger and natural conditions for human life

\section{CONCLUSION}

As a result of the research, a method of dynamic meta-anamorphosis was developed, which works at the final stage of risk controlling HIS and allows to significantly simplify and speed up the time of solving the problem of complex analysis and risk assessment in critical areas of human activity.

The method that works in real time has the following obvious advantages (Chernenkiy, V. et al, 2018):

- the reduction of dimensionality of the problem being solved for a number of local risks in critical areas of human activity minimized in the integral indicator of anamorphism;

- the possibility of calculating anamorphosis based on the integral risk index and making a decision in a homogeneous linear space of this integral index;

- anamorphism of initial values of risk indicators for any pre-defined threshold, allowing to identify those areas where the integral risk indicator is above/below a predetermined threshold; 
- the possibility of visual modeling of solutions, taking into account the local/integral risk changing in time, which allows to determine the negative / positive trends in the change of the given risks and to offer qualitative and quantitative measures to reduce them to the established values;

- identification of hidden time patterns of behavior of various parameters of the problem being solved, implicitly depending on the integral risk index;

- construction and analysis of scenarios of development of events and actions of the decision maker related to the dynamic change of the integral risk indicator in time.

Areas of further work may include research in the following fields:

- dynamic anamorphic method for visualization of big data flows and decision support, allowing to obtain and analyze scenarios of risk development in time determined by the methods of assessing the situations with which the decision maker works;

- presentation of the integral risk index in various tasks of analysis and assessment of risk indicators in the most critical areas of human activity;

- various neural network implementations algorithm dynamic meta-anamorphosis;

- further improvement of forecasting of time series of risk indicators using genetic programming methods.

- saving scenarios and decide on appropriate solutions with the aim of building subsystems situational risk management.

- create a hybrid model that combines the results of the hierarchy analysis method, which can obtain the risk rating, expressed a quantitative evaluation and the method of dynamic meta-anamorphosis which will be able to take into account the rating of the integral indicator of risk.

\section{REFERENCES}

Chernenkiy. V. et al, 2017. Using the metagraph approach for addressing RDF knowledge representation limitations. Internet Technologies and Applications (ITA), DOI: 10.1109/ITECHA.2017.8101909.

Chernenkiy, V. et al, 2018. Metagraph Approach as a Data Model for Cognitive Architecture. Advances in Intelligent Systems and Computing, Vol. 848. Springer, Cham. DOI:https://doi.org/10.1007/978-3-319-99316-4_7

Chernenkiy, Valeriy, et al., 2018. The hybrid intelligent information system approach as the basis for cognitive architecture. Procedia computer science. Sci. 145, pp. 143-152.

SIPRI Yearbook 2017: Armaments, Disarmament and International Security // Oxford University Press. Available at: https://www.sipri.org/sites/default/files/2017-09/yb17-summary-eng.pdf (accessed 20 Jule 2019)

Rajonirovanie territorij po genezisu pavodkov i navodnenij. Available at: https://geographyofrussia.com/wpcontent/uploads/2015/01/196_1.jpg (accessed 20 Jule 2019)

Skvortsov, V. et al, 2019. Feature Recognition Module of the CAPP System. In 2019 IEEE Conference of Russian Young Researchers in Electrical and Electronic Engineering (EIConRus), Moscow, Russia, pp. 1769-1772.

Skvortsova, M. et al, 2017. Hybrid Intelligent System for Risk Assessment based on Unstructured Data. Proceedings of the 2017 IEEE Russia Section Young Researchers in Electrical and Electronic Engineering Conference, pp 560-564. DOI: 10.1109/EIConRus.2017.7910616.

Skvortsova, M. and Terekhov, V, 2019. Study of The Possibility of Applying Intellectual Methods in Creating Risk Analysis Decision Support System in The Socio-Economic Sphere. In 2019 IEEE Conference of Russian Young Researchers in Electrical and Electronic Engineering (EIConRus), Moscow, Russia, pp. 1892-1896.

Skvortsova, M. and Grout, V., 2018. Basic approaches to assessing risks and threats in decision support systems. 2018 IEEE Conference of Russian Young Researchers in Electrical and Electronic Engineering. Moscow, Russia. pp. 1563-1566, DOI: 10.1109/EIConRus.2018.8317397

The Worldmapper website. Available at: https://worldmapper.org/_accessed 20 Jule 2019)

Terekhov, V. I., et al., 2019. Cognitive Visualization in Management Decision Support Problems. Optical Memory and Neural Networks Vol. 28, No. 1, pp. 27-35.

Worldmapper: The Relaunch. Available at: http://www.viewsoftheworld.net/ (accessed 20 Jule 2019) 